\title{
Nonuniform discharge currents in active plasma lenses
}

\author{
J. van Tilborg, ${ }^{1}$ S. K. Barber, ${ }^{1}$ H.-E. Tsai, ${ }^{1}$ K. K. Swanson, ${ }^{1,2}$ S. Steinke, ${ }^{1}$ C. G. R. Geddes, ${ }^{1}$ \\ A. J. Gonsalves, ${ }^{1}$ C. B. Schroeder, ${ }^{1}$ E. Esarey, ${ }^{1}$ S. S. Bulanov, ${ }^{1}$ N. A. Bobrova, ${ }^{3}$ \\ P. V. Sasorov, ${ }^{3}$ and W. P. Leemans ${ }^{1,2}$ \\ ${ }^{1}$ Lawrence Berkeley National Laboratory, University of California, Berkeley, California 94720, USA \\ ${ }^{2}$ Department of Physics, University of California, Berkeley, California 94720, USA \\ ${ }^{3}$ Keldysh Institute of Applied Mathematics, Moscow 125047, Russia
}

(Received 21 December 2016; published 24 March 2017)

\begin{abstract}
Active plasma lenses have attracted interest in novel accelerator applications due to their ability to provide large-field-gradient (short focal length), tunable, and radially symmetric focusing for charged particle beams. However, if the discharge current is not flowing uniformly as a function of radius, one can expect a radially varying field gradient as well as potential emittance degradation. We have investigated this experimentally for a 1-mm-diameter active plasma lens. The measured near-axis field gradient is approximately $35 \%$ larger than expected for a uniform current distribution, and at overfocusing currents ring-shaped electron beams are observed. These observations are explained by simulations.
\end{abstract}

DOI: 10.1103/PhysRevAccelBeams.20.032803

\section{INTRODUCTION}

Laser plasma accelerators (LPAs) [1] have produced $\mathrm{MeV}$ to multi-GeV electron beams in mm-cm scale plasma structures [2-9]. The LPA community is pursuing applications such as ultrafast electron beam pump-probe studies [10], compact light sources including coherent $\mathrm{x}$ rays [11-14] and incoherent MeV photons [15-18], and high energy particle colliders driven by multiple LPA stages $[19,20]$. For these applications, collimation and focusing of electron beams over short, $\mathrm{cm}$-scale distances is important.

When considering traditional transport elements for $\mathrm{cm}$-scale focusing, challenges will be encountered: (i) Due to the $1 / \gamma^{2}$ scaling of the focusing strength, with $\gamma$ the electron relativistic Lorentz factor, solenoids have weak focusing (long 10-m scale focal lengths) for relativistic electrons and have hence only been applied to energies of a few $\mathrm{MeV}$ or less [21]. (ii) The strong field gradients of miniature quadrupoles (of order $500 \mathrm{~T} / \mathrm{m}$ [22]) are promising, as is the more favorable $1 / \gamma$ scaling of the focusing strength, but the effective focal length is strongly increased when one considers that three lenses of varying and opposite strengths need to be combined to achieve equal focusing in both planes [23]. Thus, a triplet configuration still only enables $>10-100 \mathrm{~cm}$ focal lengths for $>100 \mathrm{MeV}$ class beams. Based on concepts revived from the 1950s ion accelerator community [24-27], recently Ref. [28] demonstrated that discharged plasma lenses [29-32] have

Published by the American Physical Society under the terms of the Creative Commons Attribution 4.0 International license. Further distribution of this work must maintain attribution to the author(s) and the published article's title, journal citation, and DOI. the potential to be of great value to compact accelerator applications, due to tunability, high-field gradients $(>3000 \mathrm{~T} / \mathrm{m})$, and radial focusing symmetry. For example, $I_{0}=600$ A for a $R=0.25 \mathrm{~mm}$ capillary yields a field gradient of $1800 \mathrm{~T} / \mathrm{m}$, corresponding to a focal length of $5 \mathrm{~cm}$ for a $L=30 \mathrm{~mm}$ long structure and $1 \mathrm{GeV}$ electrons. Here the focal length was calculated using the thin-lens approximation $f=1 /(k L)$, with $k=e(\partial B / \partial r) / m_{e} \gamma c, e$ the electronic charge, $m_{e}$ the electron rest mass, $(\partial B / \partial r)$ the magnetic field gradient, and $c$ the vacuum speed of light.

Discharge capillaries are typically described as a gasfilled elongated volume of square or circular cross section inside a glass or sapphire block, with $\mathrm{cm}$-scale length and $\mathrm{mm}$-scale diameter. By flowing a discharge current $I_{0}$ through a circular capillary of radius $R$, the current density is $J_{0}=I_{0} / \pi R^{2}$ for a uniform current distribution. Using Ampère's law $2 \pi B(r) r=\mu_{0} \int_{0}^{r} J\left(r^{\prime}\right) 2 \pi r^{\prime} d r^{\prime}$, the radial gradient $(\partial B / \partial r)$ of the azimuthal component of the magnetic field is

$$
(\partial B / \partial r)_{\text {uniform }}=\mu_{0} I_{0} /\left(2 \pi R^{2}\right)
$$

with $\mu_{0}$ the vacuum permeability, and assuming cylindrical asymmetry.

Although Eq. (1) is useful for estimating the focal length of an active plasma lens, in practice the transverse current distribution is a function of radial coordinate $r$. For a collisional plasma, the plasma conductivity $\sigma$ depends on the plasma density $n_{e}$ and the plasma temperature $T_{e}$ through [33]

$$
\sigma=\frac{32 \epsilon_{0}^{2}}{\ln \Lambda} \frac{\left(k_{B} T_{e}\right)^{3 / 2}}{e^{2} m_{e}^{1 / 2}}
$$


with $\Lambda=n_{e} \lambda_{D}^{3}, \quad \lambda_{D}=\sqrt{\epsilon_{0} k_{B} T_{e} / n_{e} e^{2}}, \epsilon_{0}$ the vacuum permittivity, and $k_{B}$ the Boltzmann constant. Due to the weak scaling of $\sigma$ with density $\left(n_{e}\right.$ only appears in the logarithmic function $\Lambda$ ), the current distribution is dominated by the plasma temperature. Thus, with the plasma near the wall typically colder than the plasma near the axis in a discharged capillary, a radial temperature gradient exists that is expected to drive a nonuniform current distribution.

In this paper the nonuniform transverse current distribution in the plasma lens is investigated. Section II describes magneto-hydrodynamics (MHD) simulations of the temperature and current distributions in the discharged capillary. Section III presents experimental results using a 1-mm-diameter active plasma lens. Conclusions are presented in Sec. IV.

\section{MODELING}

To quantify the radial current distribution, onedimensional radial MHD simulations were performed. For numerical reasons, the wall and the plasma temperature $T_{e}$ were initiated at $0.3 \mathrm{eV}$ [34]. For the simulations presented next, the parameters $I_{0}=93 \mathrm{~A}(800 \mathrm{~ns}$ after the peak of the 440 A discharge pulse), $R=500 \mu \mathrm{m}$, and He gas at $8 \times 10^{16} \mathrm{~cm}^{-3}$ atomic density were used, with the current pulse shape shown (solid black curve) in the bottom right inset of Fig. 2. The density and temperature (at $800 \mathrm{~ns}$ after the peak of the current pulse) are shown in Fig. 1(a). The plasma temperature has increased near the axis to $>3 \mathrm{eV}$, while the density is reduced on axis and increases towards the wall (the formation of a plasma channel). The current density and azimuthal magnetic field versus radius are shown as circles in Figs. 1(b) and 1(c), respectively. The MHD simulations support the previous assertion that the current density $J(r)=\sigma E \propto T_{e}^{3 / 2}$ is dominated by the temperature distribution $T_{e}(r)$; the current density is maximum on axis and falls off towards the wall. Here $E$ is the axial component of the electric field in the comoving frame in which the plasma is locally at rest.

A simple expression for the azimuthal magnetic field $B(r)$ can be derived assuming $J(r)=\sigma(r) E \propto T_{e}(r)^{3 / 2}$. Following the work of Ref. [34], the temperature distribution satisfies the heat flow equation

$$
\frac{1}{x} \frac{d}{d x}\left(x \frac{d u}{d x}\right)=-u^{3 / 7}
$$

where $T_{e}=A u^{2 / 7}$ with $A=\left(7 R^{2} E^{2} \sigma_{0} / 2 \kappa_{0}\right)^{1 / 2}, x=r / R$, and the thermal and electric conductivities were assumed to scale as $\kappa=\kappa_{0} T_{e}^{5 / 2}$ and $\sigma=\sigma_{0} T_{e}^{3 / 2}$, respectively. The boundary conditions satisfy $d T_{e}(x=0) / d x=0$ and $T_{e}(r=1)=T_{*}$ with $T_{*}$ the temperature at the wall. The current distribution can be expressed as

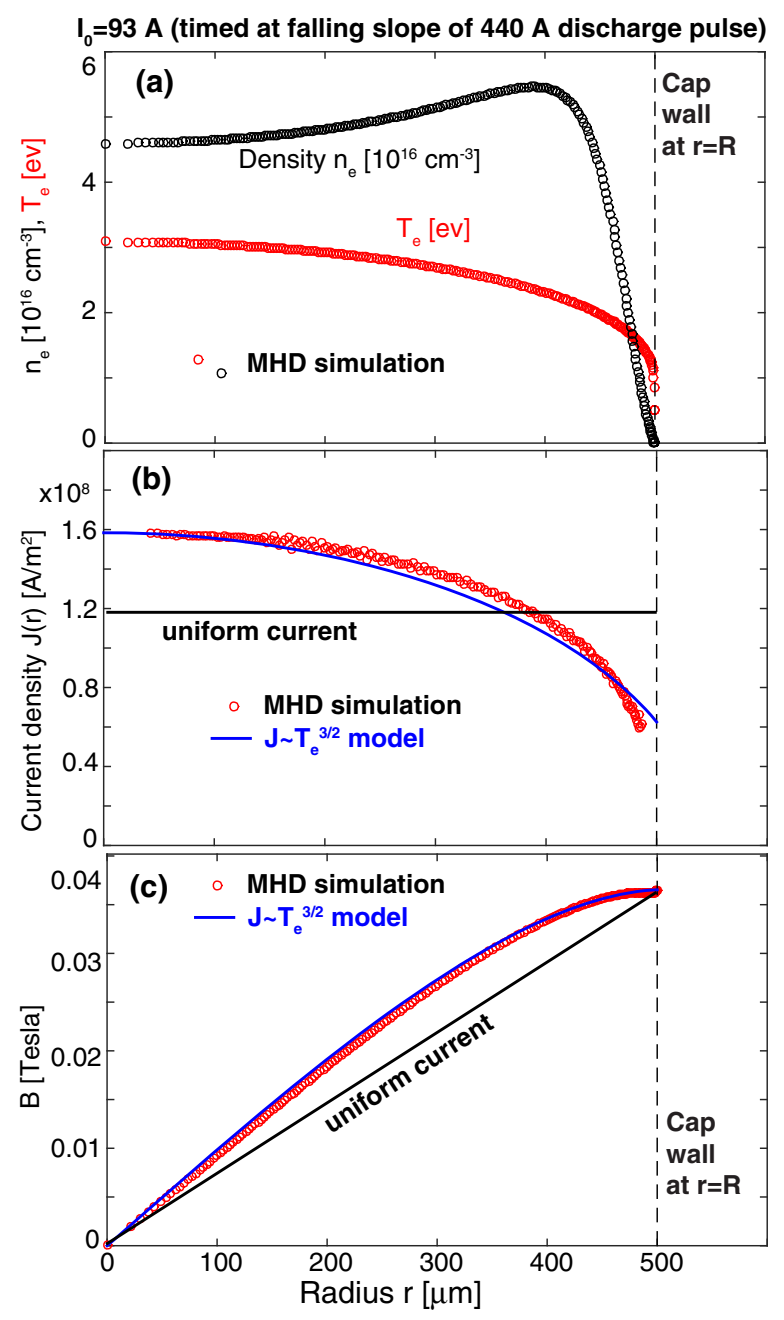

FIG. 1. MHD simulations of a 1-mm-diameter plasma lens yield the radial distribution of (a) the electron temperature and plasma density, (b) the transverse current density, and (c) the magnetic field profile. A simplified model was proposed (plotted as solid blue curves), based on $J(r) \propto T_{e}(r)^{3 / 2}$, with $T_{e}(r) \propto$ $u(r)^{2 / 7}$ as described in Ref. [34]. One can observe an approximately $35 \%$ enhancement in near-axis current density and B-field gradients.

$$
J(r)=\frac{I_{0} u(r)^{3 / 7}}{2 \pi R^{2} m_{I}}
$$

with

$$
m_{I}=\int_{0}^{1} u^{3 / 7} x d x
$$

Near the axis $x=r / R<1$,

$$
J(r)=\left(\frac{I_{0}}{\pi R^{2}}\right) \frac{u(0)^{3 / 7}}{2 m_{I}}\left[1-\frac{3 x^{2}}{28 u(0)^{4 / 7}}\right],
$$

and

$$
B(r)=\left(\frac{\mu_{0} I_{0}}{2 \pi R^{2}}\right) \frac{u(0)^{3 / 7}}{2 m_{I}} x\left[1-\frac{3 x^{2}}{56 u(0)^{4 / 7}}\right]
$$


Equation (7) predicts that the gradient near the axis is

$$
(\partial B / \partial r)_{r=0}=\left(\frac{\mu_{0} I_{0}}{2 \pi R^{2}}\right) \frac{u(0)^{3 / 7}}{2 m_{I}} .
$$

For a cold wall boundary condition $T_{*}=0$, the solution of Eq. (3) yields $u(0) \simeq 0.067, u(1)=0, m_{I} \simeq 0.106$, and $(\partial B / \partial r)_{r=0} \simeq 1.48(\partial B / \partial r)_{\text {uniform }}$.

The solid curve in Fig. 1(c) shows $B(r)$ based on this simplified model in case of a nonzero wall temperature, assuming $u(1)=0.01$, and is in good agreement with the MHD simulation [one can derive that $u(0) \simeq 0.087$ and $\left.m_{I} \simeq 0.131\right]$. It can be observed that the on-axis magnetic field gradient is larger compared to the case of a uniform current distribution. The near-axis field gradient is enhanced by approximately 35\%. In Sec. III experimental data is discussed that confirms this expected field gradient enhancement.

\section{EXPERIMENTAL OBSERVATION}

To experimentally study the role of discharge current nonuniformity in active plasma lenses, the LPA setup as depicted in Fig. 2 was operated at LBNL's BELLA Center. A $1.8 \mathrm{~J}$ laser was focused by a $2 \mathrm{~m}$ focal length parabola to a spotsize of $w_{0}=22 \mu \mathrm{m}$ onto a deLaval gas jet of $840 \mu \mathrm{m}$ diameter, tilted $30^{\circ}$ from normal. As described in Ref. [35], a razor blade on a translation stage was attached to the body of the jet, allowing for a sharp density shock profile normal to the laser beam to be present. The longitudinal profile of the density (up-ramp to shock, sharp shock-induced density drop, followed by a gentle down-ramp to vacuum), as shown in the bottom left inset in Fig. 2, was controlled with jet pressure and blade location. By flowing hydrogen gas at electron densities of $2-6 \times 10^{18} \mathrm{~cm}^{-3}$, tunable stable electron beams of energy $30-200 \mathrm{MeV}$ were produced, with the

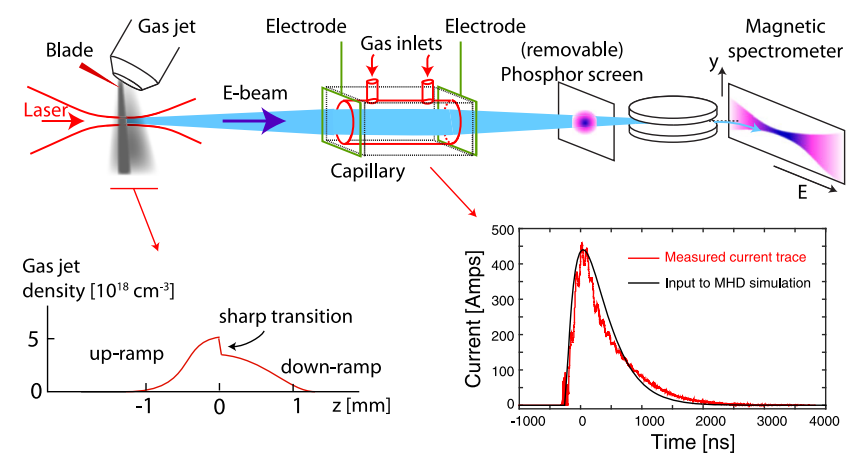

FIG. 2. Schematic concept of the experimental setup. Electron beams produced by a jet-blade assembly [35] were propagated through the plasma lens to either a transverse charge diagnostic (phosphor screen) or energy distribution diagnostic (magnetic spectrometer). The bottom left inset shows the (simplified) plasma profile at the LPA, while the bottom right inset displays the measured discharge current profile (red curve) and profile used for the MHD simulations (black curve). energy spread on the sub-10\% (rms) level. Charge was $10-50 \mathrm{pC}$ at divergence $2-7 \mathrm{mrad}$, depending on the parameters of operation. Although shot-to-shot pointing fluctuations were of order $10 \%-20 \%$ of the beam divergence, at times minute-scale pointing drifts of $<3 \mathrm{mrad}$ with respect to the laser axis occurred (under investigation).

Following a vacuum drift of $30 \mathrm{~cm}$ after the jet, the electron beams entered the active plasma lens. The lens was a sapphire-based cylindrically symmetric structure of length $L=15 \mathrm{~mm}$ and radius $R=0.5 \mathrm{~mm}$. When filled with $\mathrm{He}$ at $8 \times 10^{16} \mathrm{~cm}^{-3}$ atomic density (2.5 Torr), the plasma density $n_{e}$ was estimated to be $\simeq 5 \times 10^{16} \mathrm{~cm}^{-3}$ based on the MHD simulations in Fig. 1(a). The current trace through the capillary is shown in the bottom right inset in Fig. 2, at 440 A peak current. The timing of the LPA laser and electron beam with respect to the discharge pulse was adjustable. A phosphor screen was positioned $140 \mathrm{~cm}$ downstream of the plasma lens, and a magnetic spectrometer $240 \mathrm{~cm}$ from the plasma lens. The relevant properties of the magnetic spectrometer (energy resolution and fringe fields) are described in Ref. [36]. The imaging resolution of the phosphor screen was estimated to be $0.3 \mathrm{~mm}$ (rms). Although the beam divergence was variable over the range 2-7 mrad (rms), the angular acceptance of the capillary was $\pm 1.7 \mathrm{mrad}$.

The results of a discharge timing scan are shown in Fig. 3, taken at $8 \times 10^{16} \mathrm{~cm}^{-3}$ atomic He density in the capillary and peak current of $440 \mathrm{~A}$. The blue circles display the peak signal level on the camera (in arbitrary units), and the red circles the beam size (in units of $\mathrm{mm}$ ). The error bars are defined as the standard error of the mean. The timing was varied on the falling slope of the discharge pulse (see bottom right inset in Fig. 2). The electron energy was $62 \pm 2 \mathrm{MeV}$ with a $6 \%$ (rms) energy spread. One can observe that the smallest electron beams where recorded around $50 \mathrm{~A}$. Note that a uniform-current-density capillary

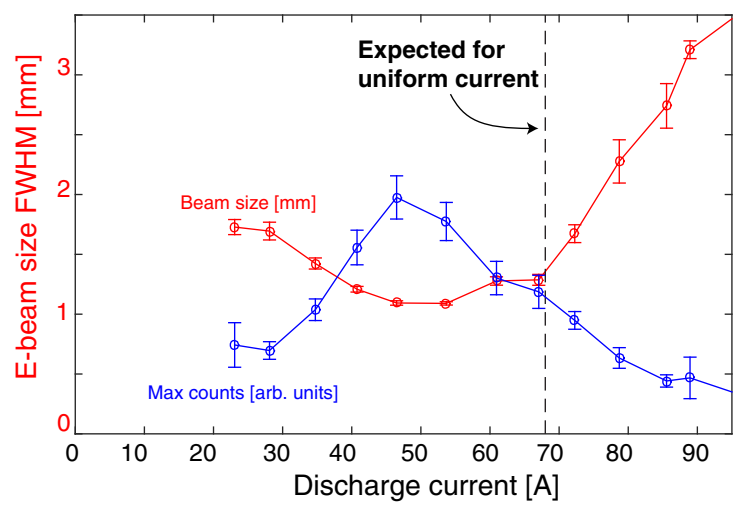

FIG. 3. Electron beam size (red circles) and peak signal level (blue circles) on the phosphor screen in a current timing scan at $8 \times 10^{16} \mathrm{~cm}^{-3} \mathrm{He}$ atomic density. The dashed black line displays the current at which one would expect focusing in case of uniform radial current distribution. 


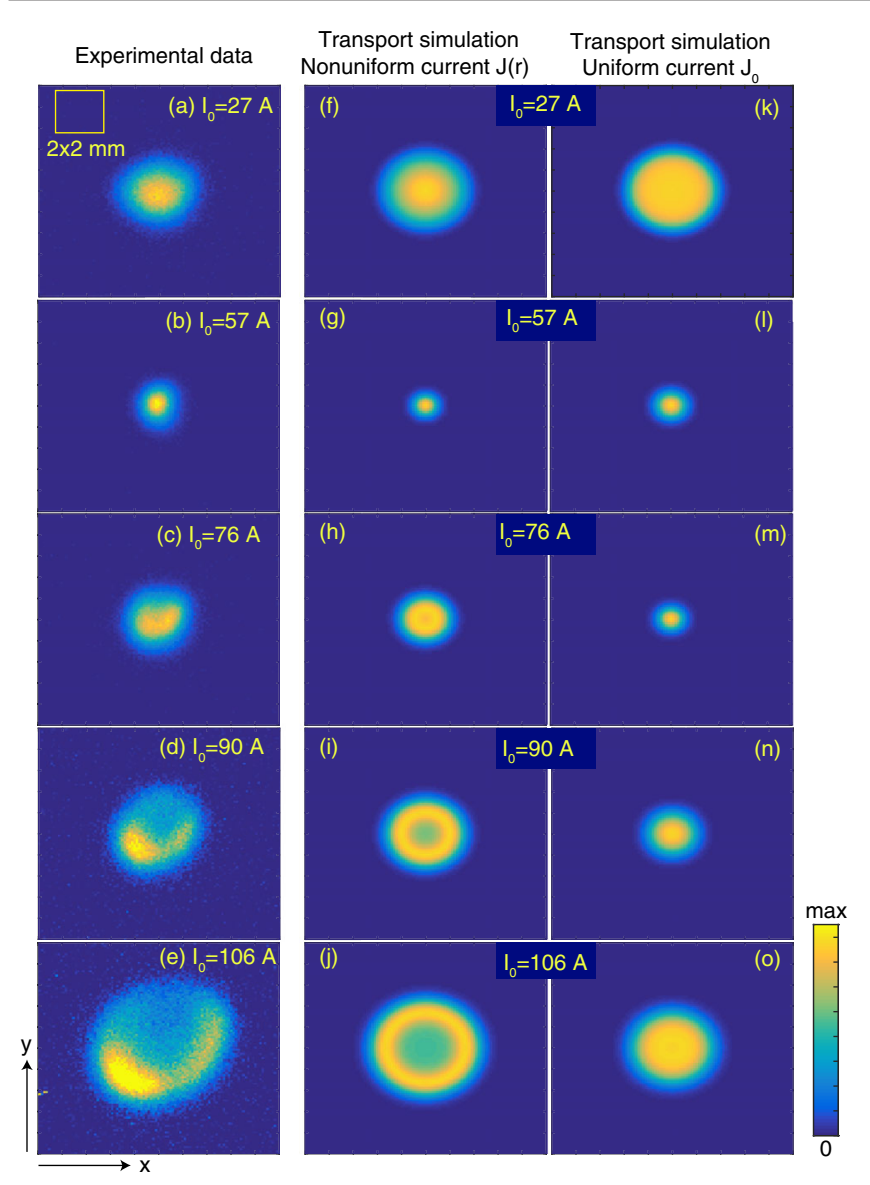

FIG. 4. Charge distribution on the phosphor screen at various discharge timings (and thus discharge currents). Experimental data in (a)-(e), simulations based on a plasma lens with nonuniform current in (f)-(j), and uniform current in (k)-(o). For overfocusing currents $(>60 \mathrm{~A}$ ), the nonuniform current simulations (h)-(j) match the observed ring-shape beam profiles, while for the underfocused current of $27 \mathrm{~A}$ the beam profile is filled in for both experiment (a) and simulation (f).

would have predicted a current of 68 A for focusing (approximately 35\% larger than measured); see dashed blue line in Fig. 3. This observation supports the assertion that current nonuniformity from near-axis heating leads to a $35 \%$ near-axis gradient enhancement; hence $35 \%$ less current is needed to achieve the $\partial B / \partial r$ gradient required for focusing.

The left column in Fig. 4 shows representative images of the charge distribution at the phosphor screen (same scan as Fig. 3). For currents above $60 \mathrm{~A}$ in Figs. 4(c)-(e), where the lens is stronger, the electron beam has overfocused and is diverging at the location of the screen. One can observe that the beam has developed a ring-shaped profile. However, at 27 A in Fig. 4(a), where the lens is weaker and the electron beam is still converging onto the screen, the electron beam is larger but without the ring-shaped profile.

To understand these features, the observations are compared to a particle tracking simulation based on the propagation of $>10^{5}$ particles from LPA source $(5 \mu \mathrm{m}$ source size was used) to phosphor screen, using the experimental conditions for the LPA and transport line described above. Space charge effects during transport and electron beam driven wakefields in the lens have been evaluated, and were not found to significantly impact the results. The particle simulations in Figs. 4(f)-(j) used the B-field profile of Fig. 1(c) in the lens, with gradient enhancement $35 \%$. One can observe that the overall features of the experimental data are reproduced. For currents $>60$ A the central part of the electron beam in the lens is overfocused, leading to a weak charge density. However, the electrons near the walls experience a weaker field gradient and have not expanded significantly. This leads to the ring-shaped beam profile. For weaker currents $<30 \mathrm{~A}$ both near-axis and near-wall electrons are still converging to the phosphor screen, maintaining a filled-in profile. When the transport simulations are repeated for a uniform current density plasma lens, the ring-shaped profile is not observed and the current needed to focus the electron beam has shifted from $52 \mathrm{~A}$ to $68 \mathrm{~A}$. Note that the mm-level electron beam size at focus, observed in experiment [Fig. 4(b)] and simulation [Fig. 4(e)], is a result of the chromaticity of the active plasma lens combined with the electron beam energy spread, as well as the imaging resolution of the phosphor screen camera. These effects were included in the tracking simulations. The profile asymmetries in Figs. 4(c)-(e) can be qualitatively explained through comparison to transport simulations by a $<3$ mrad electron beam pointing offset causing a nonuniform charge transmission through the capillary. It was also verified using transport simulations that a tilted misaligned active plasma lens does not reproduce the asymmetry.

The transport simulation results demonstrate that the current distribution parametrization based on the MHD simulations and simplified model of $J(r) \propto T(r)^{2 / 3}$, discussed in Sec. II, provides good agreement with experimental data, highlighting the role of the current density distribution as shown in Fig. 1(b). Depending on the electron beam size at the plasma lens, this current nonuniformity can manifest itself as a degradation of emittance, since the focal strength of the lens contains a radial dependence. For an electron beam confined to $<R / 2$ inside the lens, the field gradient [cf. Fig. 1(c)] is approximately constant and little emittance degradation is expected. Also, MHD simulations presented in Ref. [32] indicate that current nonuniformity is minimized at lower peak current and higher fill pressures. This could imply that operating a 440 A peak discharge pulse at $200 \mathrm{~A}$ timing (trailing edge) is more detrimental to the emittance than using a 200 A peak discharge pulse at peak timing. The benefit of higher fill pressures is that ionization takes longer to accomplish, leaving less time for (detrimental) plasma heating [32]. For a fixed nonzero wall temperature, the reduction in on-axis temperature then results in improved current uniformity. These concepts will be studied in future experiments. 


\section{CONCLUSIONS}

In summary, experiments were performed on 1-mmdiameter active plasma lenses using LPA-produced electron beams. Through comparison to transport simulations and MHD-simulated plasma lens properties, several key experimental observations were explained by the role of nonuniform radial current density (more current near the lens axis than near the wall): (1) The near-axis field gradient $\partial B / \partial r$ was enhanced, meaning less current was needed to focus the electron beam than expected for a uniformcurrent density lens. (2) For overfocusing currents, the beam developed a ring-shaped profile, while (3) at underfocused currents the beam remained with a filled-in profile. Future studies will focus on characterization, and mitigation, of emittance degradation from an active plasma lens with radially varying focusing strength.

\section{ACKNOWLEDGMENTS}

This work was supported by the U.S. Department of Energy (DOE) under Contract No. DE-AC02-05CH11231, by the National Science Foundation under Grant No. PHY1415596 , by the U.S. DOE National Nuclear Security Administration, Defense Nuclear Nonproliferation R\&D (NA22), and by the Gordon and Betty Moore Foundation under Grant ID GBMF4898.

[1] E. Esarey, C. B. Schroeder, and W. P. Leemans, Physics of laser-driven plasma-based electron accelerators, Rev. Mod. Phys. 81, 1229 (2009).

[2] J. Faure, Y. Glinec, A. Pukhov, S. Kiselev, S. Gordienko, E. Lefebvre, J.-P. Rousseau, F. Burgy, and V. Malka, A laser-plasma accelerator producing monoenergetic electron beams, Nature (London) 431, 541 (2004).

[3] C. G. R. Geddes, Cs. Toth, J. van Tilborg, E. Esarey, C. B. Schroeder, D. Bruhwiler, C. Nieter, J. Cary, and W. P. Leemans, High-quality electron beams from a laser wakefield accelerator using plasma-channel guiding, Nature (London) 431, 538 (2004).

[4] S. P. D. Mangles et al., Monoenergetic beams of relativistic electrons from intense laser-plasma interactions, Nature (London) 431, 535 (2004).

[5] W. P. Leemans, B. Nagler, A. J. Gonsalves, Cs. Tóth, K. Nakamura, C. G. R. Geddes, E. Esarey, C. B. Schroeder, and S. M. Hooker, GeV electron beams from a centimetrescale accelerator, Nat. Phys. 2, 696 (2006).

[6] H. T. Kim, K. H. Pae, H. J. Cha, I. J. Kim, T. J. Yu, J. H. Sung, S. K. Lee, T. M. Jeong, and J. Lee, Enhancement of Electron Energy to the Multi-GeV Regime by a Dual-Stage Laser-Wakefield Accelerator Pumped by Petawatt Laser Pulses, Phys. Rev. Lett. 111, 165002 (2013).

[7] X. Wang et al., Quasi-monoenergetic laser-plasma acceleration of electrons to $2 \mathrm{GeV}$, Nat. Commun. 4, 1988 (2013).

[8] W. P. Leemans et al., Multi-GeV Electron Beams from Capillary-Discharge-Guided Subpetawatt Laser Pulses in the Self-Trapping Regime, Phys. Rev. Lett. 113, 245002 (2014).

[9] W. T. Wang et al., High-Brightness High-Energy Electron Beams from a Laser Wakefield Accelerator via Energy Chirp Control, Phys. Rev. Lett. 117, 124801 (2016).

[10] I. Tudosa, C. Stamm, A. B. Kashuba, F. King, H. C. Siegmann, J. Stöhr, G. Ju, B. Lu, and D. Weller, The ultimate speed of magnetic switching in granular recording media, Nature (London) 428, 831 (2004).

[11] A. R. Maier, A. Meseck, S. Reiche, C. B. Schroeder, T. Seggebrock, and F. Grüner, Demonstration Scheme for a Laser-Plasma-Driven Free-Electron Laser, Phys. Rev. X 2, 031019 (2012).

[12] Z. Huang, Y. Ding, and C. B. Schroeder, Compact X-ray Free-Electron Laser from a Laser-Plasma Accelerator Using a Transverse-Gradient Undulator, Phys. Rev. Lett. 109, 204801 (2012).

[13] C. B. Schroeder et al., in Proceedings of the 35th International Free-Electron Laser Conference, FEL2013 (Brookhaven National Laboratory, New York, 2013).

[14] M. E. Couprie, A. Loulergue, M. Labat, R. Lehe, and V. Malka, Towards a free electron laser based on laser plasma accelerators, J. Phys. B 47, 234001 (2014).

[15] Y. Glinec et al., High-Resolution $\gamma$-Ray Radiography Produced by a Laser-Plasma Driven Electron Source, Phys. Rev. Lett. 94, 025003 (2005).

[16] S. Chen et al., MeV-Energy X Rays from Inverse Compton Scattering with Laser-Wakefield Accelerated Electrons, Phys. Rev. Lett. 110, 155003 (2013).

[17] N. D. Powers, I. Ghebregziabher, G. Golovin, C. Liu, S. Chen, S. Banerjee, J. Zhang, and D. P. Umstadter, Quasimonoenergetic and tunable X-rays from a laser-driven Compton light source, Nat. Photonics 8, 28 (2013).

[18] S. G. Rykovanov, C. G. R. Geddes, J.-L Vay, C. B. Schroeder, E. Esarey, and W. P. Leemans, Quasimonoenergetic femtosecond photon sources from Thomson Scattering using laser plasma accelerators and plasma channels, J. Phys. B 47, 234013 (2014).

[19] W. P. Leemans and E. Esarey, Laser-driven plasma-wave electron accelerators, Phys. Today 62, No. 3, 44 (2009).

[20] S. Steinke et al., Multistage coupling of independent laser-plasma accelerators, Nature (London) 530, 190 (2016).

[21] Z.-H. He, A. G. R. Thomas, B. Beaurepaire, J. A. Nees, B. Hou, V. Malka, K. Krushelnick, and J. Faure, Electron diffraction using ultrafast electron bunches from a laserwakefield accelerator at $\mathrm{kHz}$ repetition rate, Appl. Phys. Lett. 102, 064104 (2013).

[22] R. Weingartner et al., Imaging laser-wakefield-accelerated electrons using miniature magnetic quadrupole lenses, Phys. Rev. ST Accel. Beams 14, 052801 (2011).

[23] J. K. Lim, P. Frigola, G. Travish, J. B. Rosenzweig, S. G. Anderson, W. J. Brown, J. S. Jacob, C. L. Robbins, and A. M. Tremaine, Adjustable, short focal length permanentmagnet quadrupole based electron beam final focus system, Phys. Rev. ST Accel. Beams 8, 072401 (2005).

[24] E. Boggasch, J. Jacoby, H. Wahl, K.-G. Dietrich, D. H. H. Hoffmann, W. Laux, M. Elfers, C. R. Haas, V. P. Dubenkov, and A. A. Golubev, z-pinch plasma lens focusing of a heavy-ion beam, Phys. Rev. Lett. 66, 1705 (1991). 
[25] B. Autin, H. Riege, E. Boggasch, K. Frank, L. De Menna, and G. Miano, A Z-Pinch Plasma Lens for Focusing High-Energy Particles in an Accelerator, IEEE Trans. Plasma Sci. 15, 226 (1987).

[26] F. Dothan, H. Riege, E. Boggasch, and K. Frank, Dynamics of a $\mathrm{z}$ pinch for focusing high-energy charged particles, J. Appl. Phys. 62, 3585 (1987).

[27] A. A. Drozdovskii, A. A. Golubev, B. Yu. Sharkov, S. A. Drozdovskii, A. P. Kuznetsov, Yu. B. Novozhilov, P. V. Sasorov, S. M. Savin, and V. V. Yanenko, Formation of hollow heavy ion beams in plasma lens, Phys. Part. Nucl. Lett. 7, 534 (2010).

[28] J. van Tilborg et al., Active Plasma Lensing for Relativistic Laser-Plasma-Accelerated Electron Beams, Phys. Rev. Lett. 115, 184802 (2015).

[29] D. J. Spence and S. M. Hooker, Investigation of a hydrogen plasma waveguide, Phys. Rev. E 63, 015401 (2000).

[30] A. Butler, D. J. Spence, and S. M. Hooker, Guiding of HighIntensity Laser Pulses with a Hydrogen-Filled Capillary Discharge Waveguide, Phys. Rev. Lett. 89, 185003 (2002).
[31] C. McGuffey, M. Levin, T. Matsuoka, V. Chvykov, G. Kalintchenko, P. Rousseau, V. Yanovsky, A. Zigler, A. Maksimchuk, and K. Krushelnick, Guiding of 35 TW laser pulses in ablative capillary discharge waveguides, Phys. Plasmas 16, 113105 (2009).

[32] A. J. Gonsalves et al., Demonstration of a high repetition rate capillary discharge waveguide, J. Appl. Phys. 119, 033302 (2016).

[33] F. F. Chen, Introduction to Plasma Physics and Controlled Fusion: Volume 1, Plasma Physics, 2nd ed. (Springer, New York, 1984).

[34] N. A. Bobrova, A. A. Esaulov, J.-I. Sakai, P. V. Sasorov, D. J. Spence, A. Butler, S. M. Hooker, and S. V. Bulanov, Simulations of a hydrogen-filled capillary discharge waveguide, Phys. Rev. E 65, 016407 (2001).

[35] K. Swanson et al. (unpublished).

[36] K. Nakamura, W. Wan, N. Ybarrolaza, D. Syversrud, J. Wallig, and W. P. Leemans, Broadband single-shot electron spectrometer for $\mathrm{GeV}$-class laser-plasma-based accelerators, Rev. Sci. Instrum. 79, 053301 (2008). 